\title{
Is the Magnetic Field Preserved During Core Formation?
}

\author{
Brenda C. Matthews \\ University of California at Berkeley, Radio Astronomy Laboratory, 601 \\ Campbell Hall, Berkeley, CA, 94720, U.S.A. \\ Shih-Ping Lai \\ University of Maryland, Department of Astronomy, College Park, MD, \\ 20742, U.S.A. \\ Richard M. Crutcher \\ University of Illinois, Department of Astronomy, 1002 West Green \\ Street, Urbana, IL, 61801, U.S.A. \\ Christine D. Wilson \\ McMaster University, Department of Physics \& Astronomy, 1280 Main \\ St. W., Hamilton, ON, L8S 4M1, Canada
}

\begin{abstract}
We present recent JCMT and BIMA array polarimetry data of nearby star-forming regions in order to compare the core and cloudscale magnetic field geometries in two regions of Orion. The similarity of the magnetic field geometry in these cores to that of their ambient clouds is contrasted with JCMT data toward the Barnard 1 dark cloud in Perseus, which reveal a different magnetic field orientation between the majority of the cores and the surrounding cloud; each of the cores exhibits a different mean polarization position angle. We conclude that the preservation of the magnetic field geometry is better in cores formed within clouds with ordered large scale structures. In Barnard 1, the cores may quickly exhibit a different polarization pattern if they have, for example, rotation which differs from the large scale cloud motions, or a weaker component of ordered fields. This could also explain why the cores exhibit such different geometries from each other in Barnard 1.
\end{abstract}

\section{Introduction}

Polarized emission from dust in star-forming clouds and cores is the most effective tool for measuring the magnetic field geometry within these regions. Polarimetry in this regime provides a measure of plane-of-sky field geometry and samples the net contribution of polarized grains along the line of sight through a cloud. A determination of how much the field geometry differs between dense star-forming (or potentially star-forming) cores and their parent clouds has been 
hindered until recently, since typically only cores were of sufficient brightness for effective observations.

In this paper, we compare single dish data from the James Clerk Maxwell Telescope (JCMT) and interferometric data from the Berkeley-Illinois-Maryland Association (BIMA) array to determine whether the magnetic field geometry of parent clouds is inherited by cores once they have formed.

The JCMT is a $15 \mathrm{~m}$ single dish with a resolution of $14^{\prime \prime}$ at $850 \mu \mathrm{m}$. This corresponds to parsec length scales in nearby molecular clouds such as Perseus $(d \sim 330 \mathrm{pc})$ and Orion A and B $(d \sim 450 \mathrm{pc})$. Dust polarimetry maps were made using a polarimeter with the SCUBA (Submillimetre Common User Bolometer Array) camera (Greaves et al. 2003).

The BIMA array has 9 antennas with $1.3 \mathrm{~mm}$ SIS receivers. Polarization maps are made using polarization plates to alternatively sample left-circular and right-circular polarizations, and each of the four combinations needed to derive the Stokes' parameters, on each baseline. Resolutions of up to $3^{\prime \prime}$ and $1.5^{\prime \prime}$ are achievable in the $\mathrm{C}$ and $\mathrm{B}$ array configurations, respectively. Sensitivity is an important issue for interferometric polarimetry because arrays filter out emission on scales comparable to the minimum baseline measured. However, this spatial filtering has the advantage that large scale structure is not sampled by the array, which has permitted us to measure the field geometry of the MMS6 core distinctly from the filament in which it is embedded.

\section{The MMS6 core in the Orion A Filament}

OMC-3 is part of the Integral-shaped filament in Orion A. Its polarization pattern was mapped with SCUBA by Matthews, Wilson, \& Fiege (2001); a portion of this map is reproduced in Figure 1. In the region shown, the polarization position angles follow closely the axis of the filament and the polarization percentages (the ratio of polarized emission to total emission $\times 100 \%$ ) were found to systematically decrease toward the filament's axis, independent of whether a core was present. Matthews et al. (2001) therefore suggested that field geometry is the dominant source of the filament's depolarization (the decrease in polarization percentage with increasing intensity).

The helical field model of Fiege \& Pudritz (2000) predicts fragmentation of cores from filamentary clouds. Such cores would initially preserve the magnetic field geometry of the parent clouds. Our BIMA polarimetry map indicates a very similar polarization pattern on small scales to that of the large scale map in Figure 1. The vectors are well-aligned across $\sim$ two beam areas (i.e. the map is oversampled) The BIMA data are not sensitive to structures larger than approximately $40^{\prime \prime}$ in size, which means we are not picking up the filamentary emission seen in the SCUBA map. The mean orientation of the BIMA vectors is $-54^{\circ}$ with uncertainties of $\sim 10^{\circ}$, while the two vectors which are spatially coincident in the SCUBA map have a mean position angle of $-39^{\circ}$ with uncertainties $<2^{\circ}$. These values are roughly consistent.

As in most cores, we observe a decrease in polarization percentage as intensity increases. However, we do not detect polarization across the entire core; no polarization in the southern part of MMS6. 

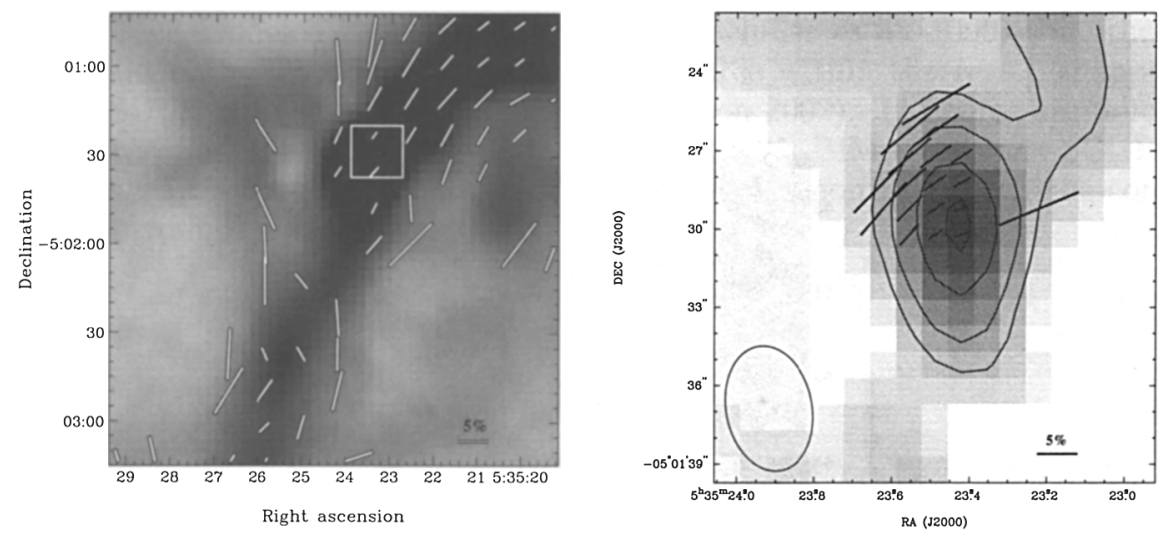

Figure 1. The left figure shows $850 \mu \mathrm{m}$ dust emission in grayscale with polarization vectors overlain. All vectors shown are $\geq 6 \sigma$ detections in polarization percentage. The vectors are binned to approximately beamwidth sampling of $5400 \mathrm{AU}$. The white box indicates the field of view of the BIMA image of the MMS6 core at right. The 1.3 $\mathrm{mm}$ intensity is shown in greyscale and contours (at levels of $6 \sigma, 12 \sigma$, $24 \sigma$ and $36 \sigma$ ). The vectors plotted are $\geq 3 \sigma$ detections in polarization percentage and spatially coincident with at least $\geq 6 \sigma$ detections in intensity. The map resolution, indicated by the oval, is $4.8^{\prime \prime} \times 3.3^{\prime \prime}$, which corresponds to $2200 \times 1500 \mathrm{AU}$ at the distance of Orion.

\section{The FIR5 core in the NGC 2024 Ridge of Orion B}

For comparison with the OMC-3/MMS6 data, Figure 2 contains the previously published maps of the NGC 2024 region of Orion B (Matthews, Fiege, \& Moriarty-Schieven 2002) and the high resolution map of its brightest core, FIR5 (Lai et al. 2002). Similarly to the Orion A data, the large scale map is not sensitive to the core emission, partly due to resolution and partly due to the depolarization effect (the lower detection limit was 1\%). Despite the negligible polarization measured at the location of FIR5 with SCUBA, Lai et al. (2002) detected strong polarization from this core with the BIMA array, as shown in Figure 2.

Comparison of the two maps shows that both exhibit a shift in vectors from east to west. However, it is clear that the shift in orientation of the position angles must occur over a very small spatial scale, less than the radius of the core. Only one vector clearly associated with the core exhibits the +ve position angle (measured $\mathrm{E}$ of $\mathrm{N}$ ) associated with the eastern edge of the ridge. Hence, the core's polarization pattern is not yet globally distinctive from that of the ambient cloud. Note however, that there is a systematic shift in polarization position angle in the BIMA data which was modeled as a curved field geometry by Lai et al. (2002). This appears to be a property of the core, as it is not evident in the large scale map. 

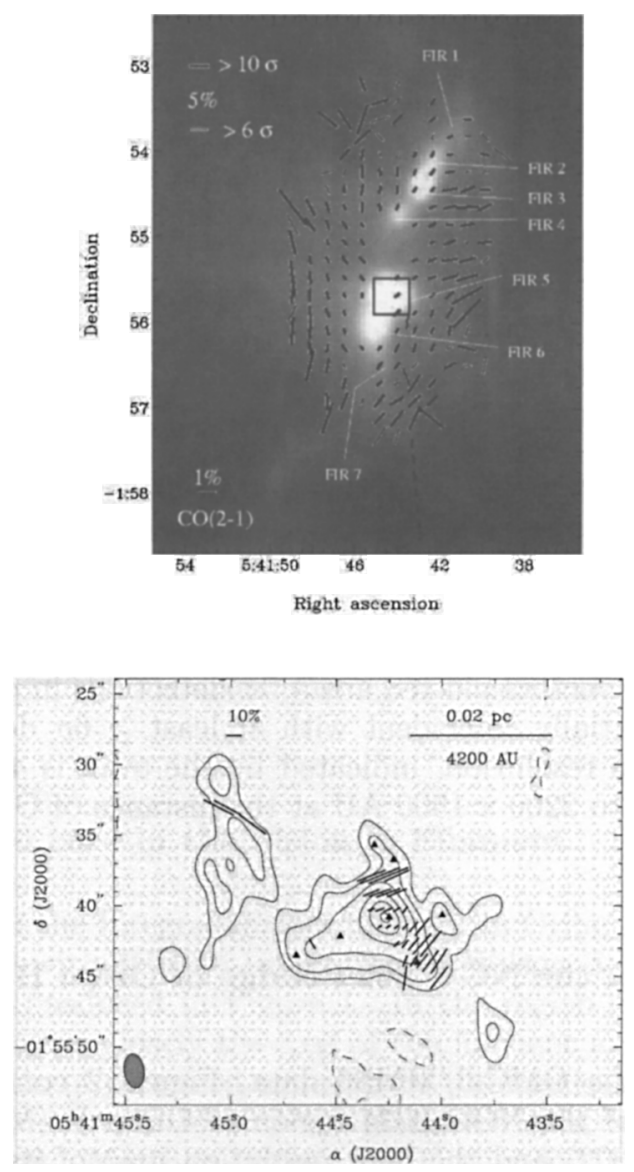

Figure 2. The SCUBA polarization map (Matthews et al. 2002) is shown on top for the entire NGC 2024 ridge of 7 cores. Note the shift of approximately $90^{\circ}$ in vector orientation between the east and west sides of the ridge. The box indicates the field of view of the BIMA map on bottom, taken from Lai et al. (2002). The BIMA data also show a shift in polarization position angle from east to west. This BIMA map is the highest resolution map made of polarized emission from dust (resolution of $1.5^{\prime \prime}$ ).

\section{The Barnard 1 Main Core}

Unlike the MMS6 in OMC-3 and FIR5 in NGC 2024, the Barnard 1 cloud in Perseus is a dark cloud without significant filamentary structure. The main 
core has been modeled as externally heated (Bachiller et al. 1990), but there are several protostellar cores and one IRAS source within the region mapped with the SCUBA polarimeter (Matthews \& Wilson 2002). The cores are unfortunately too faint to be mapped in polarimetry with BIMA, but the SCUBA data, shown in Figure 3, already reveal polarized emission from both the cores and the surrounding dust.
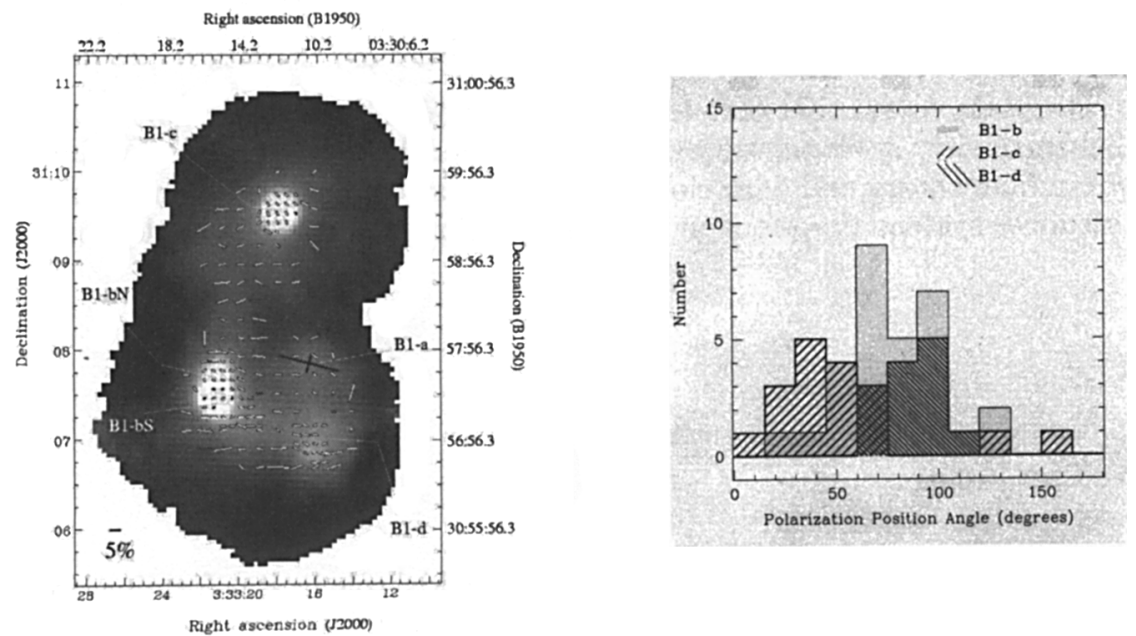

Figure 3. The SCUBA polarization map of B1 from Matthews \& Wilson (2002) is at left. All vectors are at least $6 \sigma$ detections in polarization. The polarization position angles of the cores appear obviously distinct from the surrounding, lower density, dust, which has been binned to $12^{\prime \prime}$ sampling. The core data are at half-beamwidth sampling. The histogram at right shows the polarization position angle distributions for three of the cores.

The low-intensity dust shows strong alignment in an east-west direction. This could indicate a reasonably uniform direction for the magnetic field on these scales. However, the polarized emission in cores clearly exhibits different orientations from the ambient dust, and each core's pattern is in fact distinct from the others. This is illustrated by the histogram of Figure 3, which shows the polarization position angle distribution of the three brightest cores.

\section{Discussion}

The data compiled in this paper suggest that star-forming cores formed along filaments show similar polarization patterns as their parent clouds. Data now exist in two different clouds in Orion that show that no significant tangling of 
magnetic fields appears to exist inside cores observered interferometrically which could explain the depolarization seen in single dish telescopes. Furthermore, the cores appear to retain magnetic field information from the parent cloud, at least in their envelopes after formation. Conversely, the Barnard 1 cloud contains several cores, each of which shows a different polarization position angle. It is intriguing that the more quiescent dark cloud contains cores which more quickly exhibit distinct geometries from their parent clouds. No outflow has yet been detected from the MMS6 core (Williams, Plambeck, \& Heyer 2003), and the $\mathrm{B} 1 \mathrm{c}$ outflow has also not been identified. It is possible that both these cores are pre-stellar. The B1c is also distinguished because it exhibits no polarization hole (see Matthews \& Wilson 2002 for details), unlike other starless and protostellar cores.

Our data reveal that core formation does not necessarily result in a change in field geometry in filamentary clouds. Whether the difference observed between cores in filamentary and dark clouds is due to a stronger ordered field component or stronger systemic motions in filamentary clouds has yet to be determined.

\section{The Future}

A more thorough understanding of magnetic fields' role in star forming processes clearly requires both large scale and high resolution data. Hence, we require polarimeters on sensitive cameras for single-dish telescopes, and dual-polarimetry capabilities on interferometric arrays. One constraint on measurements of large scale structure is the need to chop to remove sky noise with single dish telescopes. The SCUBA-2 camera (coming to the JCMT in 2005) will not require the telescope to chop; hence, measurements of polarized emission can be made on the scale of entire molecular clouds. This ability, combined with high sensitivity will enable us, for the first time, to study the magnetic field on cloud-scales at extinctions beyond $A_{v}=1.3 \mathrm{mag}$, which is the limit of near-infrared absorption polarimetry studies (Weintraub, Goodman, \& Akeson 1999)

The BIMA array has produced excellent maps of polarized emission from the brightest star-forming cores. However, its sensitivity is limited for weaker cores (as a comparison of Figures 1 and 2 in this paper shows). The next generation of interferometers will be able to greatly enhance the study of magnetic fields on small scales. The Submillimeter Array (SMA) is almost complete and will have dual-polarimetry capability at $345 \mathrm{GHz}$. Maps from the SMA can be combined with SCUBA data to create high resolution total polarized power maps. CARMA (Combined Array for Research in Millimeter Astronomy), the synthesis of the OVRO and BIMA arrays, will be between 2.2 and 10 times more sensitive for polarimetry than BIMA at $1.3 \mathrm{~mm}$, taking into account the increased correlator bandwidth, dual-polarimetry capability and polarimetry plates on all 15 antennas. And ALMA, although further in the future, will have multi-frequency polarimetry capabilities and the resolution to measure polarized emission from protostellar disks. 


\section{References}

Bachiller, R., Menten, K., \& del Rio-Alvarez, S. 1990, A\&A, 236,461

Chandrasekhar, S., \& Fermi, E. 1953, ApJ, 118, 113

Fiege, J., \& Pudritz, R. 2000, MNRAS, 311, 105

Greaves, J. et al. 2003 MNRAS, 340, 353

Lai, S.-P., Crutcher, R., Girart, J., \& Rao, R. 2002, ApJ, 566, 925

Matthews, B., \& Wilson, C. 2002, ApJ, 574, 822

Matthews, B., Fiege, J., \& Moriarty-Schieven, G. 2002, ApJ, 569, 304

Matthews, B., Wilson, C., \& Fiege, J. 2001, ApJ, 562, 400

Ostriker, E., Stone, J., \& Gammie, C. 2001, ApJ, 546, 980

Weintraub, D., Goodman, A., \& Akeson, R. 1999, proceedings of Protostars \& Planets IV, 247

Williams, J., Plambeck, R., \& Heyer, M. 2003, ApJ, 591, 1025 